\section{Telemedicine Applications in Disaster Medicine Mattila MAK}

Unit of Research, Kuopio University Hospital, Kuopio, Finland

"Nothing is actually more dangerous than action without decisions and know-how." In the construction of service systems for emergencies and disasters, we must start from the needs in the field. There is an obvious need to create a lead group that coordinates the actions and makes the decisions crucial for directing all of the available resources to produce the maximal benefit of input on output. Despite these self-evident principles of action, it is quite another matter to apply this theory to action. The coordination requires such experience that almost never is available at the site of an accident.

Fortunately, if the chain of information functions perfectly, such crucial actions can be directed from far away. It is the same as if the lead group is working at a distance of only hundreds meters or hundreds kilometres, provided that it is composed of the best experts available. Because the decisions and orders given by the lead group must match the actual situation at the scene, there is an obvious need for visualisation of the situation with updating video pictures from different points at the scene that use wireless methods for transmitting these images. These mobile-camera units should be directed and focused upon the most important views. The shadow areas in the standard wireless network field can be solved using transportable link-stations. Of course, the picture must be completed using verbal bidirectional communication. These elements complete the process from decisions to actions. One picture is worth of thousand words!

After completion of the action, there will be a rich data collection relative to the action. After analysis, these data can be used not only for improvement of the weak points detected, but also for training and teaching.

Currently, this quality of tele-coordination is just a dream, but it could become a reality. All of the needed technologies are available. The video, picture-based, dynamic direction of actions currently is used, but, so far, not in Disaster Medicine. As a matter of fact, management of a disaster by a lead group could be the masterpiece of tele-coordination.

Key words: coordination of resources; decision-making; disasters; disaster management; disaster medicine; telemedicine

\section{Interactive Training, Evaluation, and Testing Using Computers \\ Mattila MAK}

Unit of Research, Kuopio University Hospital, Kuopio, Finland

Interactive training is based on the accepted fact that you will learn much more by doing than by listening or reading. Performing Triage at the scene of an accident means that you are arranging all actions in correct priority order. Mastering Triage is the crucial know-how obtained from experience gained at major accidents and disasters. In case of a sudden situation, you must have a tactical preparedness model of action ready in your memory. Since tactics are an essential component for emergency action, there should be sufficient training in their applications. Triage is know-how consisting of observations, conclusions, decisions in order of importance, and actions.

Tactical simulation software follows a natural sequence of actions, using a scenario such as an accident with several casualties. The trainee leads a group that provides the medical rescue. Because one is training for leadership in making decisions and in utilisation of resources, the members of the group actually are the only human resources without ability to think or act autonomously.

Interactivity means that the course of the situation is dependent upon the trainees' decisions and actions. This interactivity is the educative component that will help the leader to learn to make rational decisions. The development of traumas is time related, but you can affect it through the application of appropriate emergency procedures. Progressive challenge is accomplished by increasing the number of casualties and keeping the resources the same. Final evaluation with detailed information of the score attained during exercises is a very important part of learning. The software can be used as well for testing individual tactical preparedness, a method for which hitherto has been a missing until now.

The principal element in the concept of computeraided training is to provide enough interactive feedback to create a tactical model in the memory. The applicable software must be available readily without a need for very powerful computers or special installations.

Key words: computer-assisted training; decision-making; disaster management; disasters; multicasualty incidents; simulation; triage

\section{Are We Better Prepared After the Estonia Experience? Murtomaa MMM \\ Ministry of Social Affairs and Health, Helsinki, Finland}

The Estonia disaster in 1994 did not constitute a heavy workload as far as patient care was concerned. Eight hospitals in three Administrative Districts in Finland accepted 70 of the 137 survivors without difficulty. There were no capacity or treatment problems. Also, the Forensic Medicine Authorities performed 94 medicolegal autopsies that provided a lot of information and new guidelines were prepared and distributed to the regional officers. Lesson learned include:

1) A massive level of interest by domestic and foreign media personnel constituted a new phenomenon for the health-care personnel that must be taken into 